\title{
Performance Evaluation of Web-Services Classification
}

\author{
A. Syed Mustafa ${ }^{1 *}$ and Y. S. Kumaraswamy ${ }^{2}$ \\ 'Department of CSE, Sathyabama University Chennai, India; syedmustafa_blr@yahoo.com \\ 2Department of MCA, Dayananda Sagar College of Engineering, Bangalore, India; yskldswamy2@yahoo.com
}

\begin{abstract}
To predict web service quality, based on quality attributes set, experiments were carried out on QWS dataset. This study investigates the efficiency of web service classifiers.
\end{abstract}

Keywords: FURIA, k Nearest Neighbor (kNN), RIDOR, Support Vector Machine (SVM), Web services, QWS dataset

\section{Introduction}

Web services garnered much attention recently. New networking technologies and applications enforces major changes in communication ${ }^{1}$. This growth, dominated by the World Wide Web application's emergence, is fueling development of various new applications in business, engineering, education, entertainment and medicine. Such applications and developments in Internet infrastructure will impact daily lives, underscoring the importance of knowing technologies, which are the base for the Internet.

Web services are considered a contemporary paradigm for developing distributed, Internet-based and platform-agnostic business applications. The appeal to the business community is due to the fact that they can interact between complicated, heterogeneous and distributed, enterprise information systems using standards for almost all interoperation aspects. Web services are software components which communicate through use of pervasive, standards-based web technologies like Hypertext Transfer Protocol (HTTP) and XML-based messaging. Web services are accessed by applications, varying in complexity from simple operations like checking a bank balance online, to complex processes like running Customer Relationship Management (CRM) or Enterprise Resource Planning (ERP) systems. As they are open standards based like HTTP and XML, including
Simple Object Access Protocol (SOAP) and Web Services Description Language (WSDL), such web services are independent of hardware, programming languages and operating systems ${ }^{2}$.

Web services are software modules that perform discrete task/tasks that are found and invoked on a network including the World Wide Web. Developers create a client application invoking many web services via Remote Procedure Calls (RPC) or through a messaging service to provide the application's logic. Web services resort to SOAP for XML payload using a transport like HTTP to carry SOAP messages to and fro. SOAP messages are $\mathrm{XML}$ documents sent between web services and calling applications. Web services are written in any language and run on any platform. A web service client is also written in any language and runs on any platform.

Web Services leverage experience in technical communities ranging from distributed systems to software engineering for example, Reference Model for Open Distributed Processing (RM-ODP). Technical/business requirements are distilled into an effective model applying it to various resources directly. Web Service model's main roles are as service providers, service consumers and service registry ${ }^{3}$. Main artifacts are services and service descriptions. An operational model is based on a find-bind-use approach. Consumers seek a service provider in a registry. After a successful search, consumers/ providers establish a connection and service delivery

${ }^{*}$ Author for correspondence 
process is undertaken. Open standards, from a technical perspective, are necessary for Web Services success. Initiatives like W3C Web Service Activity and Universal Description, Discovery and Integration (UDDI) are good examples.

QualityofService(QoS)combinesmanynon-functional characteristics. ${ }^{4}$ QoS publication selects among services with same functionality, service composition that is based on QoS and evaluates alternative execution paths for process adaptation. Also, QoS is the base for cost models driving process optimization. QoS monitoring attains customer anticipated quality levels and allows providers both to detect problems and balance cost/performance ratios. Major requirements to support QoS in Web services are ${ }^{5}$ :

Availability: the quality aspect of whether a Web service is present or ready for use immediately.

Accessibility: a service's quality aspect which represents how much it is capable of serving a Web service request.

Integrity: the quality aspect of how a Web service maintains interaction correctness regarding the source.

Performance: quality aspect of Web service, measured through throughput and latency.

Reliability: a web service's quality aspect representing the capability degree of maintaining a service and service quality.

Regulatory: quality aspect of a Web service conforming to rules, laws, compliance with standards and established service level agreement.

Security: the Web service's quality aspect of providing confidentiality and non-repudiation by authenticating parties, encrypting messages and ensuring access control.

Achieving/maintaining defined QoS properties in changing environments are a challenge for self-adapting architectures ${ }^{6}$. Service-based systems can address these challenges, as exploitation of different composition patterns (orchestration and choreography) represents achieving self-adapting architectures efficiently. Conceptually, services comprise of three levels: messages, abstract processes, and execution processes. Message level describes messages exchanged and syntax involved, with WSDL and EDIFACT being examples. Abstract processes are sequences where messages are exchanged. Execution processes implement abstract processes for execution in organizations. Business Process
Execution Language (BPEL)'s executable part is an example of standards addressing these levels ${ }^{7}$.

Web Services classification is carried out, regarding WSDL documents to be classified, in a two-step process: Keywords retrieval in WSDL documents and Documents classification.

Text mining is the computer discovering new, previously unknown information through an automatic extraction of information from large, differently, unstructured textual resources ${ }^{8}$. Text mining in web services, extracts what is relevant to a specific service category and handles accessing a Web Services description to extract category related terms. Techniques are classified into 6 sections ${ }^{9}: 1$. Runtime reconfiguration using wrappers, 2. Runtime component adaptation, 3. Composition language, 4. Workflow-driven composition techniques, 5. Ontology-driven web service composition and 6. Declarative composition.

This study uses machine learning methods like k Nearest Neighbor (kNN), FURIA, RIDOR, Support Vector Machine (SVM) classifiers to predict web service quality based on attributes. The rest of study is organized as follows: Section 2 describes in detail, quality related issues in web services. Section 3 presents the methodology followed. Section 4 presents results and discussion and Section 5 concludes the paper.

\section{Related Work}

Combination of policy and context to manage behaviors which Web services exposed during composition and in response to environment changes were discussed by Maamar et al. ${ }^{10}$, a four-layer approach being devised for this purpose and which were denoted by policy, user, Web service and resource. Behavior management and binding in this approach were under executing policies of type's permission, obligation, restriction, and dispensation. A prototype illustrating how context and policy were interwoven into Web services composition scenarios was also presented.

An architecture supporting concurrency control on Web services levels was proposed by Alrifai et al. ${ }^{11}$. An extension to standard framework for Web service transactions ensured detecting and handling transactional dependencies between concurrent business transactions. A protocol to control that could be deployed in fully distributed form within the new architecture was suggested. The proposed solutions performance was evaluated regarding throughput and response time. 
A mechanism for past Web service QoS information collection using different service users was proposed by Zheng et al. ${ }^{12}$. Based on collected QoS data, a collaborative filtering approach predicted Web service QoS values. In the end, a prototype called WSRec was implemented through Java language and deployed in the Internet to conduct real-world experiments. To study this approach's QoS value prediction accuracy, 1.5 million Web service invocation results were collected from 150 service users located in 24 countries and on 100 real-world Web services in 22 countries. The results revealed that the new algorithm ensured better prediction accuracy than other approaches. The new Web service QoS data set was then released for future research publicly.

Dynamic web service selection problems in failureprone environments, to determine a Web services subset to be invoked at run-time to ensure orchestration of composite web services was studied by Hwang et al. ${ }^{13}$. Composite and constituent web services observed that constraining sequences of invoking operations, proposing to use finite state machine to model Web service operations' permitted invocation sequences. Each execution state assigned an aggregated reliability to measure probability that given state will result in successful execution in contexts where every web service could fail with some probability. A prototype which implemented the new approach using BPEL to specify a web service's invocation order served as a test bed, to compare the proposed strategies and baseline Web service selection strategies.

A mechanism that automatically fixed robustness issues in web services and proposed by Laranjeiro et al. ${ }^{14}$. The issues were then mitigated by application of inputs verification based on defined parameter domains, including domain dependencies among parameters. This fully automated methodology improved 3 different implementations of TPC-App web services and many services available on Internet publicly. The results revealed that the new approach easily improved web services code robustness.

QoS in the context of Web Services was defined by Kritikos and Plexousakis ${ }^{15}$. Its main contribution was the analysis of requirements for semantically rich QoS-based WSDM and a precise, effective QoS-based WS Discovery (WSDi) process. Also, a road map to extend current WS standard technologies to realize semantic, functional, and QoS-based WSDi, regarding the above was presented.

Design of a CCAP, a system providing tools for adaptive service composition and provisioning was described by Sheng et al. ${ }^{16}$. A composition model was put where service context and exceptions are configurable to accommodate user's needs. Adaptive composite service's execution semantics was provided by event-driven models. The execution model was based on Linda Tuple Spaces supporting real-time and asynchronous between services communication. Three core services including coordination service, context service, and event service were implemented to schedule and execute component services automatically and were adapted to user configured exceptions and run time contexts. The new system ensured efficient and flexible support for deploying, specifying and accessing adaptive composite services. The new system's benefits were demonstrated through usability and performance studies.

A prototype framework implementation in semantic WS was presented by Zhu and Zhang ${ }^{17}$ which demonstrated the framework's feasibility by running samples of building a testing tool as a test service, composing current test services for complicated testing tasks and developing a service for test executions of WS. The framework's experimental evaluation demonstrated its scalability.

A tutorial on present XML and Web services security standards was provided by Nordbotten ${ }^{18}$ where standards include XML Signature, XML Encryption, XML Key Management Specification (XKMS), WS-Trust, WS-Security, WebServicesPolicy, WS-SecureConversation, WS-SecurityPolicy, eXtensible Access Control Markup Language (XACML), and Security Assertion Markup Language (SAML) were discussed.

A metrics suite to evaluate XML web service quality regarding its maintainability was presented by Baski and Misra ${ }^{19}$ which included: web service description language data weight, distinct message ratio metric, message entropy metric and message repetition scale metric. All proposed metrics were evaluated theoretically and validated empirically. A comparative study with similar measures proved the metric suite's worth.

A Web service framework using OWL-S advertisements, combined distinction between service and Web service of WSMO discovery framework was described and evaluated by Meditskos and Bassiliades ${ }^{20}$ where the Web service matchmaking algorithm extended objectbased matching techniques used in structural case-based reasoning, allowing 1) retrieval of Web services based on relationships and exploiting structural information of OWL ontologies and 2) exploitation of Web services 
classification in profile taxonomies and performing domain-dependent discovery. The new framework was implemented in OWLS-SLR system and then evaluated and compared to OWLS-MX matchmaker.

Generic Web services-based application session management (WS-session), two-way full duplex Web services interaction for communication, and development of Web Services Initiation Protocol (WIP) were introduced by Chou et al. ${ }^{21}$. For multimedia and voice communication-over-IP, WIP is a full-featured Web services and SOA-based communication paradigm. In the WIP SOC paradigm, every WIP communication end point is exposed as a UDDI-publishable Web resource, searchable through Web search engines with the capability of being integrated into a communication-enabled business process as services. A prototype WIP system was developed. Architectural design and system implementation of Web services-based communication were studied and applied to real converged communication services scenarios. WIP advance indicated start of a full Web service and SOAbased communication paradigm that reshaped direction of over IP communication.

FACTS, a framework for fault-tolerant composition of transactional Web services was proposed by Liu et al. ${ }^{22}$. High-level exception handling strategies were identified and a new, transactional Web services taxonomy was devised to ensure a fault-tolerant mechanism combining exception handling and transaction techniques. The authors constructed specification and verification modules to help service designers construct fault-handling logic correctly. Further, an implementation module for automatic implementation of fault-handling logic in Web Services Business Process Execution Language (WS-BPEL) was designed. A case study demonstrated the proposed framework's viability with results showing that FACTS improved composite services fault tolerance with acceptable overheads.

An Artificial Intelligence (AI) planning-based framework enabling automatic web services composition was proposed by $\mathrm{Oh}$ et al. ${ }^{23}$. Web-service compositions problem formulated regarding AI planning and network optimization problems to investigate complexity in detail. Network analysis techniques were used to analyze publicly available Web service sets. A new Web-service benchmark tool called WSBen was developed. Authors developed WSPR, a new AI planning-based heuristic Web-service composition algorithm. Extensive experiments verified WSPR against state-of-the-art AI planners.
Many large-scale evaluations on real-world web services to investigate QoS of real-world web services and provide reusable research data sets for future research was conducted by Zheng et al. ${ }^{24}$. To begin with, 21,358 web services addresses were got from the Internet after which three large-scale real-world evaluations were conducted. More than 30 million real-world web service invocations were conducted on web services in more than 80 countries by users in 30 counties in the evaluations. Detailed results were presented and comprehensive web service QoS data sets were released online publicly.

A collaborative QoS prediction approach to web services taking advantages of past web service users' usage experiences was proposed by Zheng et $\mathrm{al}^{25}$. User-collaboration concept applied to web service QoS information sharing. Next, a neighborhood-integrated approach was designed for personalized web service QoS value prediction, based on collected QoS data. Largescale real-world experiments including 1,974,675 web service invocations from 339 service users on 5,825 realworld web services was undertaken to validate the new approach. Comprehensive studies revealed that the new approach achieved higher prediction accuracy compared to other approaches. Finally, public release of the new web service QoS data set ensured valuable real-world data for future research.

\section{Methodology}

Experiments were conducted with QWS Dataset. k Nearest Neighbor (kNN), FURIA, RIDOR and SVM were classifiers which evaluated web services classification.

\subsection{QWS Dataset}

Web services ${ }^{26}$ in QWS dataset were classified into 4 categories like: 1) Platinum (high quality); 2) gold; 3) silver and 4) bronze (low quality). Classification was based on WSRF's overall quality rating. It was grouped into a specific, classification based, web service. Web services functionality helps to differentiate between various services.

Updated QWS Dataset Version 2.0 has a set of 2,507 Web services and QWS measurements conducted in March 2008 using a Web Service Broker (WSB) framework. Every row in the dataset represents a Web service and its corresponding nine QWS measurements (separated by commas). The first nine elements were QWS 
metrics measured with multiple Web service benchmark tools over six-days. QWS values represent measurements averages collected during this period. The last 2 parameters represent service name and reference to WSDL document ${ }^{27}$.

\section{2 k-Nearest Neighbor ( $\mathrm{kNN})$}

$\mathrm{k}$-Nearest Neighbor is a popular algorithm for text categorization. Many researchers found that the $\mathrm{kNN}$ achieves good performance in different data set experiments. The idea behind k-Nearest Neighbor algorithm is straightforward. When classifying a new document, a system finds $\mathrm{k}$ nearest neighbors among training documents using categories of $\mathrm{k}$ nearest neighbors to weight category candidates ${ }^{28}$. A drawback of $\mathrm{kNN}$ algorithm is its efficiency, as it compares a test document with training set samples. Also, performance of this algorithm depends on a suitable similarity function and appropriate value for parameter $\mathrm{k}$. $\mathrm{kNN}$ Classification Algorithm is as follows:

$\mathrm{k}=$ number of nearest neighbors

for each test example $\mathrm{z}=\left(\mathrm{x}^{\prime}, \mathrm{y}^{\prime}\right)$

do

compute $\mathrm{d}\left(\mathrm{x}, \mathrm{x}^{\prime}\right)$ for each $(\mathrm{x}, \mathrm{y}) \in \mathrm{D}$

select $\mathrm{D}_{\mathrm{z}} \subseteq \mathrm{D}$, the set of $\mathrm{k}$

closest training examples

$$
\mathrm{y}^{\prime}=\arg \max _{\mathrm{v}} \sum_{\left(\mathrm{x}_{i}, \mathrm{y}_{i}\right) \in \mathrm{D}_{z}} \delta\left(\mathrm{v}, \mathrm{y}_{i}\right)
$$

\subsection{Fuzzy Unordered Rule Induction Algorithm (FURIA)}

Founded on RIPPER ${ }^{29}$, Fuzzy Unordered Rule Induction Algorithm (FURIA) is a rule-based classification method which learns fuzzy rules - and not conventional rules - and unordered rule sets instead of rule lists. Its advantage is in preserving simple, comprehensible rule sets. It has modifications/extensions and uses a rule stretching method to handle uncovered examples. Experiments proved that FURIA outperformed original RIPPER and classifiers like C4.5, as regards classification accuracy greatly.

Fuzzy rules are compared to conventional rules with advantages. For example, conventional (non-fuzzy) rules produce "sharp" decision boundary models and abrupt transitions among classes which are not intuitive. Also, one anticipates support for a class provided rule to reduce from "full" (inside rule core) to "zero" (near boundary) slowly and not immediately. "Soft" boundaries, is a Fuzzy rule characteristic, but convert to crisp boundaries when classification decisions are planned. Fuzzy rules have flexible boundaries, an example being to use aggregation operators to combine fuzzy rules that are not axis-parallel.

Conventional rule learners lead to a decision list producing rules learned by turn for all classes starting from smallest (regarding relative occurrence frequency) and ending with the second largest. A default rule is added to majority class, and new query instance is classified by the list's first rule that covers it.

This leads to both advantages and disadvantages. For example, it could have an unwanted bias as classes are not treated asymmetrically. Comprehensibility is compromised by sorting rules on a priority basis (rules condition part includes negated earlier rule conditions). FURIA learns an unordered rule set to offset this. So, a rule set for every class in a one-versus-rest scheme where the resulting model is incomplete, i.e., a new query can be uncovered by a rule (hence, decision lists faces less problems).

\subsection{RIDOR}

RIDOR is RIpple-DOwn Rule learner that generates a default rule first and exceptions for it with least (weighted) error rate later. It generates "best" exceptions for all exceptions iterating it till it is pure. It performs a treelike exceptions expansion with the leaf having only default rules without exceptions. Exceptions are rules set that predict a class other than class in default rules. IREP detects exceptions ${ }^{30}$.

\subsection{Support Vector Machine (SVM)}

Support Vector Machine (SVM) ${ }^{31}$ is the technique of machine learning method which is modified by various researchers for different applications like classification, feature extraction, clustering, data reduction and regression in different disciplines of engineering. SVM is based on concept of Hyperplane which is built for separation of data into two classes in simple binary classification of linear separable training data vector $\left(x_{1}, x_{2}, \ldots . ., x_{n}\right)$ in $n$ dimensional space. A class decision function associated with Hyperplane is weighted sum of training data set and bias is symbolized as in equation (1):

$$
y(x)=w^{T} \phi(x)+b
$$


where ' $w$ ' is the weight vector normal to Hyperplane and ' $b$ ' is the bias value. New test data based on the classification by SVM classifier is assigned to a class depending on sign of decision function as in equation (2):

Testing data can be either belongs to class-1if

$$
w^{T} \phi(x)+b \geq 1
$$

Else if

$$
w^{T} \phi(x)+b \leq-1
$$

else

$$
w^{T} \phi(x)+b=0
$$

Support vectors can be attained by maximizing the distance between closest training points and corresponding Hyperplane. Also by maximizing the margin defined as $M=\frac{2}{|w|}$ and as minimization of $\frac{\left\|w^{2}\right\|}{2}$ under the methodologies $y_{i}\left(w^{T} \phi\left(x_{i}\right)+b\right) \geq 1$. Different number of mathematical algorithms exists for determining the value of weight and bias.

Kernel function is used to define the inner product of training data points in high dimensional feature space. The function $\phi$ maps the vector to a high dimensional space. Thus the kernel function is defined as in equation (5):

$$
k\left(x_{i}, x_{j}\right)=\phi^{T}\left(x_{i}\right) \phi^{T}\left(x_{j}\right)
$$

Some advantages of kernel function are: it reduces the mathematical and computational complexity in higher dimensional feature space. Some commonly used kernel functions are linear, polynomial, radial Gaussian and sigmoid are defined in equations (6,7 and 8):

$$
\begin{gathered}
k\left(x_{i}, x_{j}\right)=x_{i} x_{j} \text { (Linear kernel Function) } \\
k\left(x_{i}, x_{j}\right)=\left(x_{i} x_{j}+c\right)^{d}
\end{gathered}
$$

For $d \geq 2$ and $\mathrm{c}>0$ (Polynomial Kernel Function)

$$
k\left(x_{i}, x_{j}\right)=e^{\frac{\left|x_{i}-x_{j}\right|^{2}}{2 \sigma^{2}}}
$$

For $\sigma>0$ (Radial Gaussian kernel function) where $\sigma$ is the kernel function parameter.

\section{Results}

Experiments were conducted with QWS Dataset. The classifiers $k$ Nearest Neighbor (kNN), FURIA, RIDOR and SVM were evaluated for web services classification. The results are given in Table 1 and Figure 1 to 4 .
Table 1. Experiment results

\begin{tabular}{llllc}
\hline Techniques & KNN & FURIA & RIDOR & $\begin{array}{c}\text { SVM with } \\
\text { SMO training }\end{array}$ \\
\hline Classification & 76.16 & 80.55 & 78.63 & 81.37 \\
accuracy & & & & \\
Precision & 0.7625 & 0.8056 & 0.7868 & 0.8159 \\
Recall & 0.7608 & 0.8053 & 0.7864 & 0.8142 \\
RMSE & 0.2978 & 0.2762 & 0.2912 & 0.2714 \\
\hline
\end{tabular}

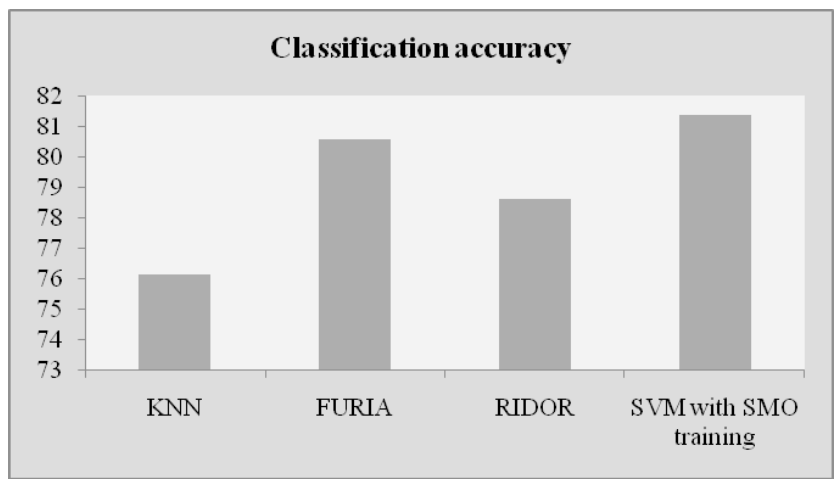

Figure 1. Classification accuracy.

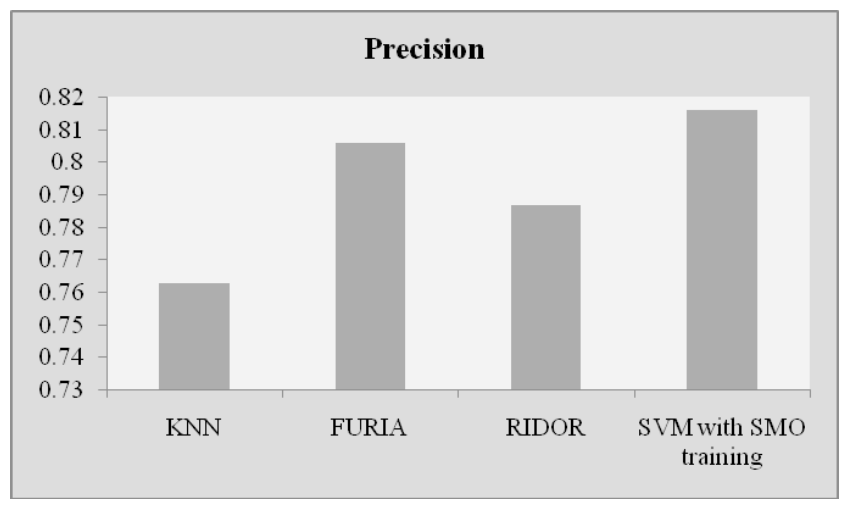

Figure 2. Precision.

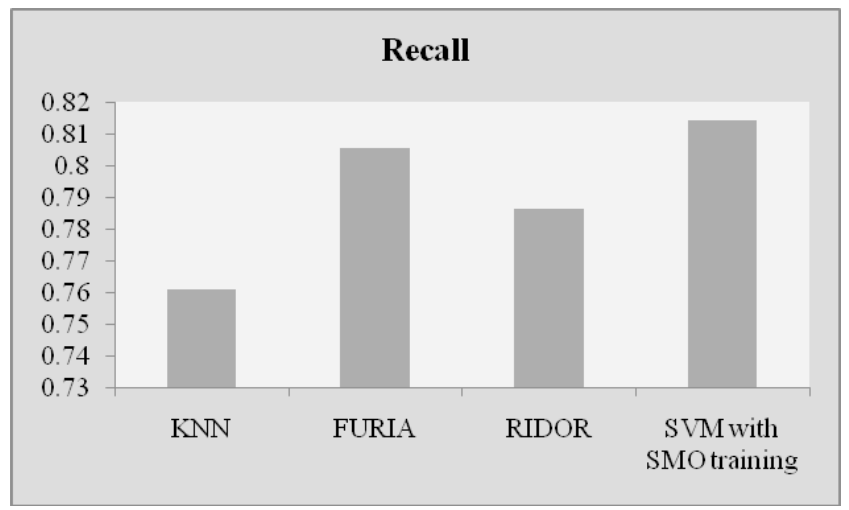

Figure 3. Recall. 


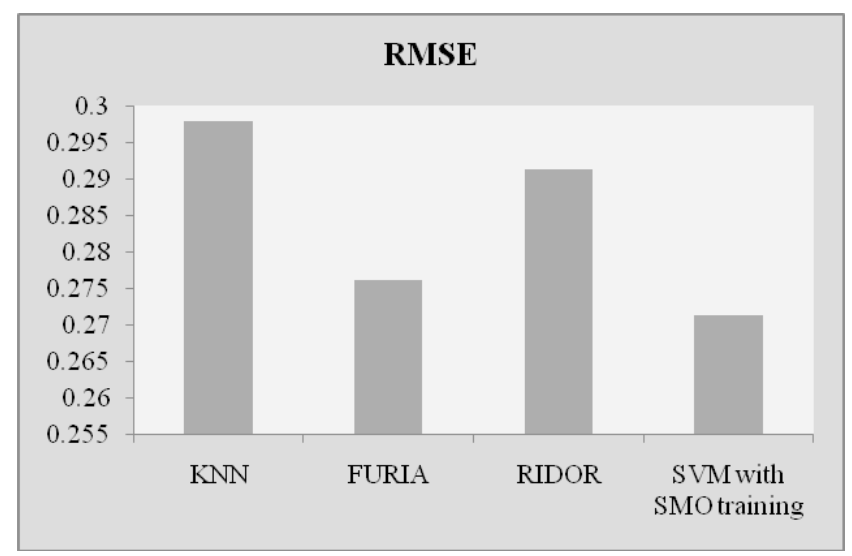

Figure 4. RMSE.

The classification accuracy is improved with SVMSMO training by $6.84 \%$ when compared with $\mathrm{kNN}$ and by $3.48 \%$ when compared with RIDOR.

Precision is improved with SVM- SMO training by 7\% when compared with $\mathrm{kNN}$ and by $3.7 \%$ when compared with RIDOR.

Recall is improved with SVM- SMO training by 7.02\% when compared with $\mathrm{kNN}$ and by $3.54 \%$ when compared with RIDOR.

RMSE is decreased in SVM- SMO training by $8.87 \%$ when compared with $\mathrm{kNN}$ and by $6.8 \%$ when compared with RIDOR.

\section{Conclusion}

With web services proliferating, QoS is a major factor to differentiate web services and providers. In selecting a web service, its non-functional properties should be considered to satisfy user's requirement constraints. This study presents web services quality prediction models, which consider non-functional properties. Experiments are carried out on QWS dataset using different classifiers like $\mathrm{kNN}$ and Naïve Bayes in this study. The average accuracy results of various classifiers are seen to be high.

\section{References}

1. Sethu H. Emerging Internet Technologies. Available from: http://www-gateway.vpr.drexel.edu/files/NewEh/htmls/ sethu.pdf

2. Cavanaugh E. Web services: Benefits, challenges, and a unique, visual development solution. A white paper published by Altova, Inc. USA. 2006.

3. Piccinelli G, Finkelstein A, Nentwich C. Web services need consistency. OOPSLA 2002 Workshop on Object-Oriented Web Services; 2002.
4. Garcia DZG, De Toledo MBF. Achieving autonomic web service integration: A quality of service policybased approach. International Transactions on Systems Science and Applications. 2007; 3:45-63.

5. Mani A, Nagarajan A. Understanding quality of service for Web services. 2005.

6. Calinescu R, Grunske L, Kwiatkowska M, Mirandola R, Tamburrelli G. Dynamic QoS management and optimization in service-based systems. IEEE Transactions on Software Engineering. 2011; 37(3):387-409.

7. Tewoldeberhan TW, Verbraeck A. Using Web Services and Artificial Intelligence Techniques to Develop Simulation Models of Business Networks. Proceedings the 15th European Simulation Symposium. 2003.

8. Shailja. Classifying web services with and without association rules. 2009.

9. Alamri A, Eid M, El Saddik A. Classification of the stateof-the-art dynamic web services composition techniques. International Journal of Web and Grid Services. 2006; 2(2):148-66.

10. Maamar Z, Benslimane D, Mostefaoui GK, Subramanian S, Mahmoud QH. Toward behavioral web services using policies. Systems, Man and Cybernetics, Part A: IEEE Transactions on Systems and Humans. 2008; 38(6):1312-24.

11. Alrifai M, Dolog P, Balke WT, Nejdl W. Distributed management of concurrent web service transactions. IEEE Transactions on Services Computing. 2009; 2(4) :289-302.

12. Zheng Z, Ma H, Lyu MR, King I. QoS-aware Web service recommendation by collaborative filtering. IEEE Transactions on Services Computing. 2011; 4(2):140-52.

13. Hwang SY, Lim EP, Lee CH, Chen CH. Dynamic web service selection for reliable web service composition. IEEE Transactions on Services Computing. 2008;1(2):104-16.

14. Laranjeiro N, Vieira M, Madeira H. A Technique for Deploying Robust Web Services. 2012.

15. Kritikos K, Plexousakis D. Requirements for QoS-based Web service description and discovery. IEEE Transactions on Services Computing. 2009; 2(4):320-37.

16. Sheng QZ, Benatallah B, Maamar Z, Ngu AH. Configurable composition and adaptive provisioning of web services. IEEE Transactions on Services Computing. 2009; 2(1): 34-49.

17. Zhu H, Zhang Y. Collaborative testing of web services. IEEE Transactions on Services Computing. 2012; 5(1):116-30.

18. Nordbotten NA. XML and web services security standards. IEEE Communications Surveys \& Tutorials. 2009; 11(3):4-21.

19. Baski D, Misra S. Metrics suite for maintainability of extensible markup language Web Services. Software, IET. 2011; 5(3):320-41. 
20. Meditskos G, Bassiliades N. Structural and role-oriented web service discovery with taxonomies in OWL-S. IEEE Transactions on Knowledge and Data Engineering. 2010; 22(2):278-90.

21. Chou W, Li L, Liu F. Web services for communication over IP. IEEE Communications Magazine. 2008; 46(3):136-43.

22. Liu A, Li Q, Huang L, Xiao M. Facts: A framework for faulttolerant composition of transactional web services. IEEE Transactions on Services Computing. 2010; 3(1):46-59.

23. Oh SC, Lee D, Kumara SR. Effective web service composition in diverse and large-scale service networks. IEEE Transactions on Services Computing. 2008; 1(1):15-32.

24. Zheng Z, Zhang Y, Lyu M. Investigating QoS of Real-World Web Services. 2012.

25. Zheng Z, Ma H, Lyu MR, King I. Collaborative web service qos prediction via neighborhood integrated matrix factorization. IEEE Transactions on Services Computing. 2013; 6(3):289-99.

26. Mohanty R, Ravi V, Patra MR. Classification of Web Services Using Bayesian Network. J Software Eng Appl. 2012; 5:291.

27. Vaadaala IV, Rao RR. Classification of Web Services Using JForty Eight.

28. Li B, Yu S, Lu Q. An improved k-nearest neighbor algorithm for text categorization. arXiv preprint cs/0306099. 2003

29. Hühn J, Hüllermeier E. FURIA: an algorithm for unordered fuzzy rule induction. Data Min Knowl Discov. 2009; 19(3):293-319.

30. Novakovic J, Minic M, Veljovic A. Genetic Search for Feature Selection in Rule Induction Algorithms.

31. Tripathy. Gender Classification from ECG Signal Analysis using Least Square Support Vector Machine. 2012. 